\title{
Les biographies anciennes d'Euripide
}

Marie Delcourt

Citer ce document / Cite this document :

Delcourt Marie. Les biographies anciennes d'Euripide. In: L'antiquité classique, Tome 2, fasc. 2, 1933. pp. 271-290; doi : 10.3406/antiq.1933.3692

http://www.persee.fr/doc/antiq_0770-2817_1933_num_2_2_3692

Document généré le 24/01/2017 


\title{
BIOGRAPHIES ANCIENNES \\ D'EURIPIDE
}

\author{
par Marie Delcourt
}

Depuis que nous lisons de longs fragments de la "Vie d'Euripide " de Satyros, nous pouvons poser mieux la question du rapport des différentes biographies entre elles. Nous sommes mieux renseignés pour voir de quoi se compose le "genos" des manuscrits et à quelle tradition se rattachent les notices de Suidas-Moschopoulos et de Thomas Magister.

Essayons de donner une description sommaire de chacun des éiêments qui composent cette tradition. Nous appellerons "genos ", puur abréger, la biographie des manuscrits et nous le citerons d'appès l'édition de M. Méridier. Nous citerons les biographies de Thomas Magister, Suidas et Moschopoulos d'après Westermann.

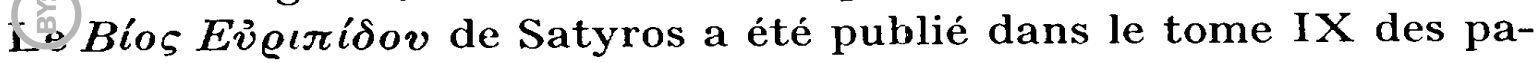
p.rus d'Oxyrhynque et dans le Supplementum Euripideum d'Arnim (Kleine Texte, Bonn, 1913).

\section{Le Genos des manuscrits.}

M. Méridier a très bien marqué que ce texte se divise en trois parties : 1-49, 49-113, 114-131.

I. Le premier morceau est une biographie complète.

II. Le second est une étude littéraire et anecdotique contenant plusieurs citations. Les histoires et les mots dont elle est faite se retrouvent souvent dans Satyros qui en donne soit l'équivalent, soit une réplique.

III. Le troisième est une notice biographique courte et excellente. L'auteur s'efforce visiblement d'isoler, dans les renseignements qu'il a sous les yeux, l'apport des poètes comiques. Le caractère d'Euripide est présenté d'une façon assez romantique : hautain, 
isolé, supérieur à la foule. Aucune allusion à ses malheurs conjugaux. Une phrase dont on voit mal la portée exacte : "son absence d'ambition lui fit autant de tort que Sophocle en tirait d'avantage ». Socrate n'est pas nommé. Les dialogues et les prologues sont critiqués assez sévèrement; tout le reste ne mérite que louanges, surtout les parties chantées. Renseignements littéraires très intéressants : 92 drames, 67 conservés, plus les trois contestés, 8 drames satyriques dont un contesté, 5 victoires.

Le renseignement des 5 victoires n'est pas dans Genos $I$; pas davantage le chiffre des 8 drames satyriques. Le chiffre des ouvres conservées est différent d'un morceau à l'autre : 78 dont 3 apocryphes, dit $I ; 67$ plus trois apocryphes, dit III, ce qui fait 70 dont 8 drames satyriques, desquels un est contesté. Il me paraît difficile, dans ce dernier passage, d'ajouter les 8 drames satyriques aux 70 autres pour arriver au total de 78 pièces conservées. Le rédacteur de cette troisième notice, semble-t-il, admet que les 67 drames conservés se répartissent comme ceci : 62 tragédies, moins 2 apocryphes, 8 drames satyriques, moins un apocryphe.

On ne voit pas pourquoi il faudrait à tout prix rendre concordants les renseignements concernant les pièces conservées. Si nous trouvons un chiffre variable d'après les époques, ce pourrait être une garantie de l'esprit critique du biographe, qui, au lieu de recopier d'après sa source le chiffre des ouvres sauvées, referait le compte d'après les manuscrits qu'il a sous les yeux. Or, précisément, l'auteur de la notice Genos III paraît avoir eu de l'esprit critique. Pourquoi ne pas admettre qu'il a eu entre les mains 70 drames d'Euripide, parmi lesquels il y en avait quatre dont il contestait l'authenticité?

Genos III est un texte qui semble avoir été écrit beaucoup plus tard que Genos $I$, à une époque où l'on ne s'intéressait plus aux potins littéraires, où la critique se plaisait moins au jeu des citations, et, devenant scolaire, cherchait à formuler des idées générales sous une forme que des écoliers pussent aisément apprendre par cour.

\section{Suidas-Moschopoulos.}

Ce texte est intéressant à cause des renseignements qu'il contient et qui ne se trouvent pas ailleurs. Cela ne veut du reste pas dire 
que ces renseignements soient tous bons. L'auteur de la notice, comme celui de la troisième du Genos, réagit contre la tendance à ridiculiser le poète en répandant les médisances des comiques.

Il dit que Philochore avait "démontré" l'origine noble de la mère d'Euripide (probablement par des arguments indirects, comme la présence d'Euripide aux fêtes d'Apollon Délien, rapportée par Théophraste). Seul avec Nicolas de Damas, il a conservé la tradition d'un exil des parents et d'un rapport avec la Béotie. L'histoire de la mort tragique d'Euripide en Macédoine se trouve partout. Mais ce biographe est le seul qui développe le "roman macédonien " et en donne plusieurs versions : Euripide aurait péri en Macédoine victime des intrigues de deux poètes, Arribée de Macédoine et Crateuas de Thessalie aidés par un esclave du roi nommé Lysimaque qui, pour sa peine, reçut dix mines. Ou encore Euripide aurait été assassiné par des femmes comme il se rendait chez Crateros le favori d'Archélaos ou chez la femme de Nicodicos. Ces développements romanesques sont brodés autour d'une tradition qu'il ne faut peut-être pas rejeter à priori: l'hostilité des Macédoniens contre le poète étranger. Addée de Macédoine, qui fleurit vers 320 , s'efforce de laver ses compatriotes du reproche d'assassinat, en disant dans une épigramme (Anth. Pal. VII, 51) que c'est la vieillesse qui fit mourir Euripide. Addée fait allusion àl a légende qui veut qu'Euripide soit mort "à cause d'une femme ", ce qui semble bien nous ramener à la même source que la notice de Suidas.

Au surplus, il faut remarquer que l'épigramme d'Addée répond à une épigramme d'Ion (Anthol.Pal. VII, 44), qui déplore le destin d'Euripide mangé par des chiens. Qu'il s'agisse d'Ion d'Ephèse, comme le veut Bergk, ou d'Ion de Samos, comme le veut Wilamowitz, l'épigramme est d'un contemporain d'Euripide et l'histoire de sa mort tragique remonte donc très haut.

Du reste, le rédacteur de la notice de Suidas n'a aucun esprit critique. S'il mentionne la démonstration de Philochore, c'est qu'elle cadre avec l'idée qu'il veut inculquer.Il raconte sans aucune précaution, sans même le traditionnel $q \alpha \sigma i$, les histoires les plus douteuses, naissance d'Euripide à Salamine le jour même de la bataille, études de peinture. Il dit qu'Euripide, de philosophe, est devenu poète tragique à cause des dangers qu'il voyait courir à Anaxagore, ce qui prouve qu'il connaît mal la chronologie littéraire du ve siècle. 
Pour ce qui est du chiffre des pièces, il rapporte ceci qui, pris littéralement, n'a aucun sens : "Euripide a écrit 75 drames selon les uns, 92 selon les autres, 77 sont conservés ». Il est impossible d'interpréter avec certitude cette phrase absurde. Dieterich, dans son article sur Euripide dans Pauly-Wissowa, entend: "il a écrit 92 drames; on en a conservé 77 ou $75 \%$. Je crois qu'il faut bien se garder de toucher à l'ordre des renseignements transmis. Le premier doit être relatif à l'époque où écrit l'auteur et l'ensemble signifie probablement: "nous connaissons encore 75 titres de pièces d'Euripide, les critiques alexandrins parlent de 92 drames et en lisaient 77 ». Il est impossible de conjecturer d'après cette notice le nombre des ouvres qu'on lisait encore à l'époque de la rédaction. Tout ce que nous pouvons constater, c'est que le chiffre de 77 drames nous ramène à peu près à Genos $I$. La notice donne ensuite le chiffre de 5 victoires dont une posthume; cette dernière indication doit venir d'une didascalie perdue pour nous des Bacchantes ou d'Iphigénie à Aulis. Notre auteur nous dit aussi qu'Euripide concourut 22 fois. Cela ferait 88 drames. Nous savons que deux drames au moins, Andromaque et Archélaos, ne furent pas joués à Athènes. Rien ne nous empêche de supposer qu'il y en eut encore deux autres qui ne furent pas présentés aux concours tétralogiques et nous voilà ramenés au chiffre de 92 . Il ne semble pas qu'il faille décompter des 92 les pièces apocryphes. Lorsqu'une œuvre étrangère s'est glissée dans le corpus, c'est très probablement à la faveur d'une homonymie, en déplaçant une ouvre authentique, comme c'est le cas pour Rhésus.

La notice de Suidas est donc une biographie sans appréciation littéraire, dans le genre de Genos $I$, mais le roman macédonien y tient une place démesurée (11 lignes sur 33).

\section{Thomas Magister.}

Cette notice est faite à peu près comme Genos $11 I$, mais pour un public qui n'est pas athénien, car l'auteur croit devoir ajouter que Salamine est une île de l'Attique. Thomas Magister semble avoir puisé à peu près tous ses éléments dans une source analogueà celle de Genos I, mais il les fait entrer dans une composition plus soignée. La mention des tableaux d'Euripide vient après le nombre des tragédies, comme si l'on avait voulu récapituler toute l'activité du 
poète. La brève appréciation littéraire (Genos 7-9) est développée en un éloge qui mérite d'être cité, tant il est intelligent. L'auteur s'adresse visiblement à un public qui a une culture philosophique :

"Prompt d'esprit comme personne et se donnant tout entier au travail, Euripide se tourna vers l'art dramatique et il y brilla noblement. Il fit dans cet art des inventions incomparables. Façonner le sujet dès l'entrée dans le drame et s'emparer pour ainsi dire de l'auditeur afin de le faire progresser : c'est l'art propre d'Euripide. Ce poète est remarquable par la clarté et la simplicité des mots dont il se sert, par sa façon de varier l'exposé grâce à des argumentations, à un rythme agréable, à l'introduction de pensées bien enchaînées entre elles et bien liées au sujet ».

Les renseignements que Thomas Magister donne indépendamment de Genos $I$ sont : la mention des 5 victoires et des 8 drames satyriques; l'anecdote de Céphisophon qui, ici, est acteur; comme chez Suidas-Moschopoulos, ce sont les déboires conjugaux d'Euripide qui le déterminent à quitter Athènes ; - enfin, la citation de Philémon et celle d'Alexandre d'Étolie, rapportée à Aristophane, qui devaient figurer dans beaucoup de Vies d'Euripide comme "l'Égypte, présent du Nil ", dans presque tous nos livres d'histoire.

Comme l'auteur de Genos III, Thomas Magister évite de nommer Socrate parmi les maîtres et amis d'Euripide. Dans Genos III, on trouve cités Archélaos et Anaxagore; ici, ce sont Anaxagore et Prodicus. L'omission paraît bien volontaire, mais on ne voit pas comment on pourrait l'expliquer.

\section{La notice D'Aulu-Gelle (Nuits attigues, XV, 20)}

Il faut dire un mot du chapitre des Nuits attiques qui est consacré à Euripide. Nous verrons plus loin qu'on a voulu y trouver un texte apparenté à la première partie du Genos et à la notice de Suidas. En réalité, le chapitre d'Aulu-Gelle se distingue au milieu de tous les autres textes parce qu'il est le seul qui soit purement biographique. Aucune remarque, ni sur les ouvres, ni sur les qualités du poète. Genos $I$ et Suidas, qui ne contiennent pas d'appréciation littéraire, donnent au moins des chiffres et des titres de pièces. Rien de semblable dans la notice d'Aulu-Gelle. Celle-ci est de plus assez malveillante. Elle est seule à mettre sous l'autorité de Théopompe l'histoire du commerce d'herbes de la mère d'Euripide. Du reste, 
il est visible qu'elle remonte à une biographie qui développait particulièrement tout ce qui était relatif à l'enfance du poète. Elle est seule à nous dire qu'Euripide commença d'écrire à 18 ans. Elle est seule aussi à nous donner des détails sur l'erreur commise par le père qui, sur la foi d'un oracle mal compris, orienta d'abord son fils vers les concours sportifs. Il est difficile de savoir si AuluGelle trouva dans la source qu'il consultait les circonstances précises dont il entoure cette anecdote (ce sont des Chaldéens qui ont rendu l'oracle; les jeux où Euripide est vainqueur sont les Thesea et les Eleusinia): il est très capable de les avoir inventées lui-même. Pour ce qui est du fond, je crois que l'historiette ne mérite aucunc créance: elle doit avoir été tirée tout entière des passages où Euripide attaque les athlètes. On se méfie des renseignements qu'Aulu-Gelle est seul à donner, lorsqu'on voit comment il explique la sévérité d'Euripide à l'égard des femmes. C'est, dit-il, ou bien qu'il ne supportait pas leur commerce, ou bien qu'il en eut deux en même temps, "comme c'était chez les Athéniens chose admise et légale ". Au lecteur de choisir l'une de ces explications, que le narrateur paraît trouver également admissibles. Il est visible qu'il ne sera arrêté par aucune des gloses que les scholiastes tirent des passages mêmes qu'ils doivent expliquer. Cela rend sceptique à propos d'un renseignement qui n'a rien de suspect en soi : les honneurs exceptionnels rendus par les Macédoniens au tombeau et leur refus de restituer aux Athéniens le corps du poète mort.

La notice des Nuits Attiques a le mérite de nous avoir conservé les trois vers d'Alexandre d'Étolie sur le contraste entre l'abord difficile d'Euripide et la douceur de ce qu'il écrivait. Nous essaierons de montrer ci-dessous que ces vers expliquent comment s'est fabriquée l'histoire de la "mauvaise haleine", dont nous trouvons une version dans Genos III et une autre dans Satyros.

\section{LA "Vie d'Euripide» de Satyros.}

Ce qui nous reste de cette biographie nous permet du moins de voir à quel genre littéraire elle appartient. Elle ne ressemble nullement aux résumés scolaires que nous avons reconnus dans la fin du Genos, dans les notices de Suidas-Moschopoulos et de Thomas Magister.

En revanche, elle est étroitement apparentée aux deux premières parties du Cienos et surtout à la seconde. 
Dès lors, on peut se demander si Satyros a servi de source aux parties du Genos qui donnent les mêmes renseignements que lui.

Leo a étudié la Vie de Satyros dans les Goettinger Nachrichlen de 1912. Il estime (p. 273 sqq.) que cette Vie est la source du Genos des manuscrits. L'auteur de ce Genos aurait traité l'étude de Satyros comme Héraclide Iemhos avait traité les Vies des philosophes du même Satyros, en y ajoutant assez librement des renseignements dûs à Philochore et à d'autres.

M. Frey a repris la question dans une thèse de Zurich $\left(^{1}\right)$. Il a montré d'une manière convaincante qu'il ne faut pas poser la question comme si le Genos faisait un seul bloc. Il divise ce texte et aboutit pour les parties qu'il y distingue aux conclusions suivantes :

"Les parties du Genos 1-44 ou 49 et 62-69 (éd. Méridier) forment un ensemble, apparenté aux Vies d'Euripide de Suidas et d'AuluGelle. Tout cela ne remonte pas à un texte unique, mais à une compilation dont la date approximative est donnée par les noms d'Eratosthène, Alexandre d'Étolie et Hermippe ( $\left.{ }^{2}\right)$. L'origine hybride du texte se trahit dans le ton et dans la tendance qui n'ont aucune unité. On y trouve tantôt de l'admiration, tantôt de la malveillance.

"Au contraire, Satyros a inspiré directement et peut-être uniquement les morceaux 49-61, 91-113 et peut-être aussi 114-135 ».

Telle est la thèse de M. Frey. Nous croyons que Satyros et Genos II ont une source commune, et qu'on ne peut rien affirmer de plus. Tout d'abord, disons que nous ne découperons pas comme lui le Genos des manuscrits.

Laissons d'abord de côté ce que nous avons appelé Genos III (114-135) qui n'a aucun rapport avec Satyros. C'est une notice où l'on entrevoit un Euripide d'un caractère'bien tranché, conforme à

(1) Der Bíos Ev̉@ıríov des Satyros und seine literaturgeschichtliche Bedeutung, Gotha, Perthes (1919).

La découverte du papyrus a provoqué la publication de plusieurs études plus ou moins approfondies. Outre celles de Frey et de Leo, citons :

Maas, Satyros, Berl. philol. Wochenschr., 1912, pp. 1075 sqq. - Richards, Satyrus, Life of Euripides, Class. Rev., 1913, pp. 47 sq. - M. L. de Courten, Satiro il biografo di Euripide, Atene e Roma, 1915, pp. 122 sqq. - Gerstinger, Satyros Bíos Ev̉@ıríov, Wiener Studien, 1916, pp. 54 sqq.

(2) Il signale avec raison que la note 1.85 peut aussi bien venir d'une Vie de Denys que d'une Vie d'Euripide. 
ce que nous appelons l'esthétique romantique. Elle ne rappelle ni la forme, ni la méthode de Satyros. On pourra objecter qu'un arrangeur, passant du dialogue à l'exposé, devait forcément changer de ton. Mais ce qui caractérise Satyros et qui manque totalement ici, c'est, comme Leo l'a très bien vu, qu'il fait uniquement de l'interprétation de passages, de la critique à coups de citations. L'idée part-elle de la citation ou bien vient-elle y aboutir? I e critique invente-t-il l'explication pour placer une citation, ou bien, à la fin d'un exposé, trouve-t-il dans sa mémoire la citation qui s'y ajuste exactement? Il est difficile d'établir avec certitude la méthode d'un texte si morcelé, mais quelques anecdotes ont été évidemment déduites de passages d'Euripide; et il semble bien que tout l'ouvrage a été composé de cette manière. Ce qui est sùr, c'est que ce jeu de citations lancées et glosées ne pouvait plaire qu'à un public très cultivé, qui lisait encore des pièces nombreuses d'Euripide : chaque vers rappelé devait éveiller en lui des échos. Il était peu exigeant quant à la qualité des anas qu'on lui rapportait : les historiettes de Satyros apparaissent souvent, dès qu'on les dégage de leur appareil littéraire, d'une qualité aussi basse que celles qui remplissent la seconde partie du Genos.

M Frey voudrait rattacher les lignes 62 à 90 à la première partie du Genos parce que ce passage (depuis $q \alpha \sigma i \delta \grave{\varepsilon} \alpha \dot{v} \tau \dot{\partial} \nu$ jusqu'à $\gamma \lambda v \varkappa v i \tau \varepsilon \varrho o \nu \sigma \tau o ́ \mu \alpha)$ ne se retrouve pas textuellement chez Satyros. En fait, toutes les anecdotes qui le composent ont chez Satyros une version un peu différente, mais analogue, comme on le verra ci-dessous. Seules les lignes 68-80 (les deux mariages du poète, expérience douloureuse d'où il tire sa pièce d'Hippolytc voilé, conspiration des femmes contre lui, collaboration de Céphisophon) manquent chez Satyros. Si nous avions le texte complet de Satyros, on pourrait peut-être, avec précaution, déduire quelque chose de cette omission. Mais qui nous dit qu'une anecdote réplique du Genos ne se trouvait pas dans un des passages perdus de la Vie?

Notre Genos $I I$ est donc tout entier apparenté aux textes de Satyros. L'a-t-il directement utilisé? Si oui, il a laissé de côté bien des détails intéressants. Mais une omission ne prouve rien. M. Frey a raison d'attirer l'attention sur une divergence d'où peut venir un indice: le Genos, racontant l'histoire de Céphisophon, dit (1. 94) qu'Euripide lui laissa sa femme parce qu'il désirait l'avoir: 


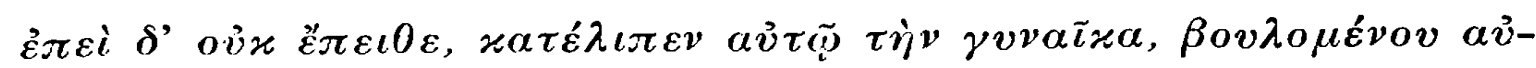
$\tau \dot{\eta} \nu \ddot{\varepsilon}_{\chi \varepsilon \iota \nu} \tau o \tilde{v} K \eta \varphi \iota \sigma o \varphi \tilde{\omega} \nu \tau o \varsigma$. C'est une redite et une platitude, et même si l'auteur n'avait pas reculé devant cela, il aurait pu

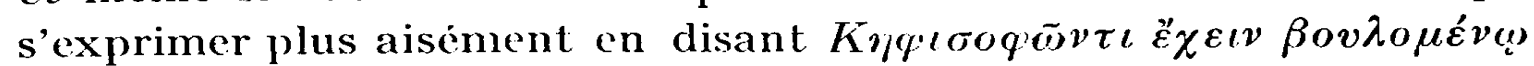

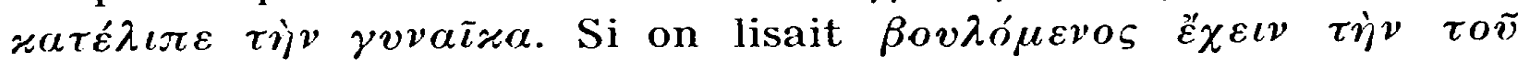

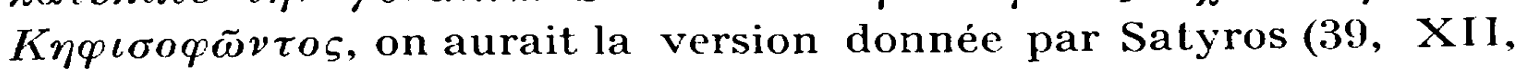
1) qui raconte que les deux hommes échangèrent leurs femmes, ou qu'ils mirent en commun celle d'Euripide remariée à Céphisophon. Cela a un sens plus digne de la comédie contemporaine que d'Euripide, mais enfin cela a un sens. 11 semble donc, dit M. Frey, qu'entre Satyros et notre Genos il y a eu un intermédiaire où l'histoire se trouvait sous une forme abrégée que notre Genos a mal comprise. C'est bien possible. Au surplus, l'anecdote - qu'elle ait eu primitivement une ou deux versions — a été certainement tirée du $\sigma v \zeta \tilde{\eta} \nu$ d'Aristophane, dans les vers qui sont cités pour l'étayer.

Ce que la Vie de Satyros a de plus caractéristique, c'est que sa partie subsistante ne contient pas un seul renseignement qui paraisse venir d'une source biographique. Les anecdotes qu'on y trouve ont leur source dans des textes littéraires : conspiration des femmes, rapports avec Céphisophon, poètes préférés à Euripide, raisons de son départ pour la Macédoine. Lorsque Satyros apporte un renseignement dont l'origine littéraire nous échappe, il est de bien mauvaise qualité : par exemple que Cléon intenta à Euripide un procès d'impiété, que le poète vieillit en Macédoine et composa le prologue des Perses de Timothée. M. Frey voit dans la Vie, comme dans le Genos, l'influence de Philochore qui a réagi contre les calomnies et le dénigrement des comiques. Il est possible que Satyros ait connu Philochore, mais il ne s'intéressait nullement à ce qui intéressait Philochore. Celui-ci est un historien de la littérature qui prouve des filiations, qui établit des dates et des chiffres. Satyros est un professeur qui étudie Euripide comme Villemain ou Nisard étudient Molière, se souciant fort peu de la date d'une édition, mais connaissant bien l'ouvre et ne voulant connaître qu'elle. Leo a très bien vu cela. Dans sa Griechisch-Römische Biographic écrite avant la découverte de notre papyrus, il disait (p. 24) que Satyros est de ceux qui aiment mieux inventer une histoire que de rester court. Dans son étude des Goettinger Nachrichten (p. 288) il insiste sur le fait que Satyros, dans notre texte, ne cite personne, sinon Euripide lui-même et des poètes comiques. 
Ainsi, nous avons, au moins en partie, les passages où Satyros s'occupe des relations d'Euripide avec Anaxagore et Socrate. Dans le Genos, il en est également question, comme d'un élément de la biographie d'Euripide et le fait s'enrichit de légendes: Euripide cuisinant une pièce avec Socrate (Genos I), renon çant à la philosophie à la suite du procès d'Anaxagore (Suidas). Satyros cherche uniquement des passages qui attestent des parentés doctrinales entre Euripide et les philosophes. Il semble même alléguer pour cela $(37$, II, 19) un fragment de Pirithoüs, pièce que toute l'antiquité considéra comme apocryphe. Assurément, des corrections et des adjonctions peuvent se trouver dans la partie perdue, (Satyros avait peut-être ses raisons de tenir la pièce authentique) mais c'est peu probable, car les fragments qui nous restent permettent de juger de la méthode : notre homme est très capable de s'appuyer sur un passage douteux, s'il sert sa thèse.

La partie du Genos qui est apparentée à Satyros se découpe d'un seul morceau et va, nous l'avons dit, de 49 à 114. Ce qui est plus curieux et qui n'a pas été remarqué, c'est que la correspondance est réciproque : le morceau qui, dans Satyros, est apparenté à Genos II s'isole parfaitement; il va de 39 , VII, 30 (citation de Philémon) à 39, XXXI (histoire des chiens thraces). Les anecdotes et surtout les citations sont plus nombreuses dans Satyros. Il arrive que la même idée soit illustrée, ici et là, par des citations différentes, mais le parallelisme est parfait, comme on le verra lorsque nous arriverons à ce passage.

Essayons maintenant de voir comment est composée la Vie d'Euripide, en tenant compte de l'importance des lacunes, ce que M. Frey n'a pas toujours fait assez soigneusement. Assurément, on ne peut avoir la prétention de retrouver le plan d'un ouvrage qui nous est arrivé dans un tel état de mutilation, mais il est assez aisé, en parcourant les fragments conservés, de trouver et de suivre des fils conducteurs.

Fragm. 8. Grandeur et hauteur du caractère d'Euripide. Satyros y reviendra à plusieurs reprises dans sa biographie qui est pleine d'admiration.

Fragm. 37. (Longo sed proximus intervallo). Euripide a été influencé par Anaxagore, mais il a dépassé l'enseignement de ce maître et il est arrivé à un spiritualisme théiste dont Satyros cite une profession. Elle frappera Clément d'Alexandrie qui l'a lue, 
du reste, dans un texte assez différent Cette position est si semblable à celle de Socrate qu'on se demande si Satyros n'a pas fait le rapprochement et si le nom de Socrate n'est pas cité dans la partie perdue, au bas de la première, en haut de la deuxième colonne du fragment 38. Rien n'empêche de le croire: Hunt estime qu'une colonne complète avait $5 \bar{a}$ à 57 lignes; il y a donc 25 à 27 lignes perdues à la fin de la colonne 1 , et $\mathbf{5}$ lignes perdues au début de la seconde. Les lignes sont très courtes (11 lettres), mais la méthode du dialogue permet de tourner court et d'abréger les transitions.

En vrai philosophe, Euripide méprise les richesses. Viennent quatre citations, qui doivent signifier: "je n'ai pour ami que l'homme de bien" - "nulle richesse ne peut suppléer à la valeur absente»- "les hommes se donnent pour s'enrichir une peine exagérée»-_. "Une femme qui vient de loin, m'apportât-elle tout l'or de l'Istros et du Bosphore, je n'en voudrais pas ». Ici, visiblement, l'idée dévie. L'on pourrait être tenté de croire que Satyros s'est servi d'une anthologie, où les fragments se trouvaient détachés de leur contexte. Mais trois fragments sur quatre ne se retrouvent pas ailleurs, et Plutarque, qui connaît le second, le donne sous une forme toute différente. Satyros connaissait bien les textes, mais il s'aidait probablement de fiches et la dernière, qu'il aurait dû classer au mot $\theta \dot{v} \varrho \alpha \theta \varepsilon v$, il l'aura classée à $\chi \varrho v \sigma o \tilde{v} \nu$. Il va sans dire que le classement peut être mental aussi bien que matériel. Bien des gens citent encore comme cela : ils évoquent un passage à la faveur d'un mot qui rappelle le contexte où on l'insère, sans se rendre compte que ce mot, dans le fragment, est tout à fait accessoire.

Fragm. 39. De même que des analogies de doctrine avaient amené l'allusion à Anaxagore, de même le nom de Socrate est amené de l'intérieur, par l'opinion sur les richesses, sur le regard pénétrant des dieux, sur le bon gouvernement de la cité.

Fragm. 39, 4. I ci, le fil casse. Il manque environ 30 lignes soit à peu près 330 lettres pour raccorder ces considérations à ce qui paraît bien être un fragment de la comédie raillant la faiblesse et l'inconséquence de la foule :

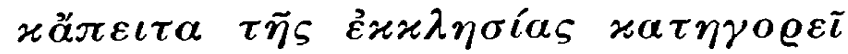

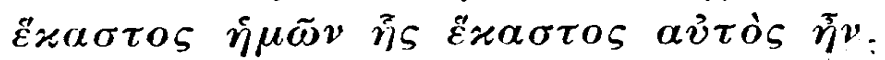


Qu'y avait-il dans la lacune? probablement un blâme très dur d'Euripide contre la foule, que Satyros mettait au point par une

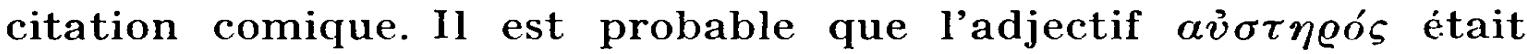
appliqué au poète, ce qui explique l'interruption de Diodora: "Voilà bien de la sévérité et du sens politique, chez les poètes comiques également"; et Satyros reprend : "Oui ; ce qui est sûr,

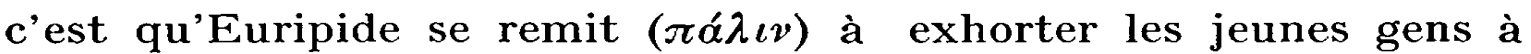
l'énergie en leur donnant comme modèle le mordant des Spartiates ».

Près de 30 lignes de lacune entre 4 et 5 ; près de 35 lignes perdues entre 5 et 6 . Impossible de nous rendre compte du lien qu'il y avait entre les fragments comiques et les opinions d'Euripide. Les citations sont tellement brèves qu'on se demande si elles ne devaient pas servir simplement d'aide-mémoire, le premier vers évoquant les suivants dans le souvenir des lecteurs lettrés.

Dans 39, 6, nous retrouvons des vers d'Euripide, mais après ceux-ci, qui flétrissent le cynisme des jeunes ambitieux :

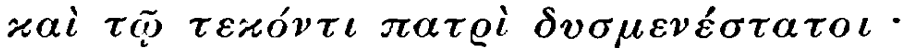

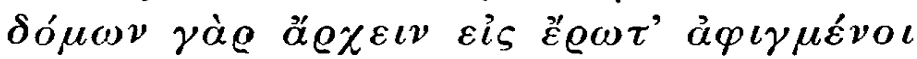

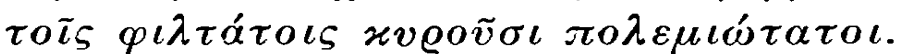

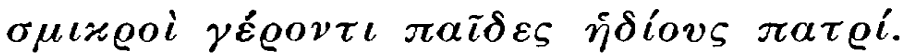

$2 \delta \delta^{\mu} \mu \omega \nu$ corr. d'Arnim pour $\delta \varkappa \mu \omega \nu$

Il est évident que le quatrième vers ne se rattache pas aux trois premiers. Comment expliquer sa présence à leur suite? Peut-être avons-nous ici une citation abrégée, qui donne d'un texte le début et le dernier vers. On peut s'imaginer un raccord dans ce genre-ci : " et le père en souffre, car ce fils lui est né dans sa vieillesse, et, à un vieillard, les enfants petits sont plus chers que les autres". Peut-être aussi le quatrième vers est-il interpolé. En effet, le jugement qui suit, sur la mauvaise éducation des temps actuels, se rattache parfaitement aux trois premiers vers, mais non au quatrième. Et l'on voit assez bien comment l'interpolation se serait produite. Après avoir lu ceci : "C'est au père qui les a engendrés qu'ils sont le plus hostiles. Car à partir du moment où ils sont possédés par le désir de gouverner la maison, leur plus grand ennemi, c'est ce qu'ils ont de plus proche ", un lecteur, réfléchissant à $\varphi \iota \lambda \tau a ́ \tau o \iota s$, aura pris ailleurs le vers suivant, sur la prédilection 
des pères pour les enfants petits, qui se rattache à un tout autre ordre d'idées.

Après une appréciation sur l'art d'Euripide, inspirée d'Aristophane, vient tout le paquet d'anecdotes, de citations et de mots qui se retrouve dans Genos $I I$. Ce paquet se découpe de la façon la plus nette. Du reste, l'ordre suivi n'est pas le même dans les deux textes et Satyros est plus abondant que Genos $I I$. Voici le détail des concordances :

Vie 39, VIII, 30 sqq., équivalent de Genos 109-113. L'admiration de Philémon est attestée par une citation différente de ce poète. Satyros donnait de plus une réponse (perdue) d'Euripide analogue à une riposte de Démosthène abrégée du Contre Aristogiton $(\mathrm{I}, 40)$.

Vie, 39, IX, équivaut à Genos 62-68.

L'histoire de la caverne est identique; Satyros en tire une conclusion sur la grandeur solitaire d'Euripide, le Genos sur l'origine de ses métaphores marines. Ils terminent pareillement par des citations différentes d'Aristophane. La citation du Genos n'est pas d'Aristophane, mais l'auteur la donne comme de ce comique.

Vie, 39, X, 1-22, passage propre à Satyros. Cette fois, au lieu d'une anecdote isolée, on a tout un morceau bien composé. Les hommes, dit l'auteur, reprochaient à Euripide d'être d'un abord difficile; les femmes, de les avoir maltraitées dans ses ouvres. Preuve du premier fait: il fut accusé d'impiété par Cléon. Preuve du second - et à partir de la ligne 22, nous retrouvons l'anecdote de Genos 100-104, racontée en termes presque identiques - : la conspiration des femmes contre Euripide qu'elles épargnent au nom des Muses.

Vie, 39, XI correspond à Genos 104-108.

Le revirement d'Euripide à l'égard des femmes est attesté dans chaque texte par une citation de Mélanippe enchaînée qu'un papyrus de Berlin a permis de rattacher à un seul et même discours ( ${ }^{1}$ ). Le choix des deux passages est tout à fait caractéristique de la méthode des deux auteurs. Celui du Genos, prend l'idée la plus générale: "les hommes ont tort de toujours blàner les femmes; elles valent mieux qu'eux ». Satyros donne des exemples particuliers: "Sans femme, une maison ne va pas. Ce sont des femmes

(1) Supplementum Euripideum, p. 31 sqq. 
qui à Delphes et à Dodone, interprètent la pensée des dieux ". Il goûte les idées curieuses qui portent si bien la marque d'Euripide, que nous lui attribuerions le fragment presque à coup sûr s'il nous était transmis sans signature.

Vie 39, XII correspond à Genos 91-93.

Satyros introduit l'anecdote de Céphisophon par deux passages des Fêtes de Cérès, qui sont cités, non par le personnage principal, mais par l'une des interlocutrices, qui prendra encore la parole dans la suite de la conversation.

Vie 39, XIII correspond à Genos 93-96 et 91.

Suite de l'histoire de Céphisophon, à qui Euripide cède sa femme ; dans Satyros, il semble qu'il y a échange des deux femmes (le début est mutilé) ou peut-être partage. Vient ensuite, dans la Vie, une intervention de Diodora qui demande pourquoi, devant une faute commise par une femme, on accuse uniquement celle-ci et non son complice. A l'appui de son dire, elle invoque l'autorité de Socrate et un passage de la République (p. $455 \mathrm{~d}$ ), sur l'équivalence morale des deux sexes. L'histoire d'Hystaspe qui suit (39, $X I V)$ se rattache certainement au même contexte: défense des femmes, ou, plus exactement ici, d'une femme qui devait se trouver accusée, comme Andromaque par Hermione, d'user de sortilèges pour capter l'amour d'un homme.

Le même sujet occupe le début de la colonne XV. L'ne brusque interruption ramène au sujet. C'est la haine de ses concitoyens qui a amené Euripide à s'exiler. Des poètes sans talent lui ont été préférés: Acestor, Dorilaos, Morsimos, Mélanthios. L'interlocutrice s'étonne: jamais elle n'a entendu les noms de ces poètes...

Après une lacune de vingt lignes environ, vient (XVI)' une critique évidemment malveillante rapportée d'après un des ennemis d'Euripide. On recommande à celui-ci de ne pas confondre la plaisanterie et le bavardage. La dernière ligne intelligible est la trentième, où se trouvent ces mots "l'hiver suivant, d'autres... "

Puis (XVII), on nous rapporte l'histoire d'une parodic malveillante ou d'une interruption pendant une représentation d'Ino. Ino est une pièce antérieure aux Acharniens et si Satyros met l'anecdote en rapport avec l'exil d'Euripide en 408, c'est qu'il ignorait totalement la chronologie de son auteur. Mais le passage est trop mutilé pour qu'on puisse en conclure grand'chose. Vingt lignes environ - 220 lettres -... sont perdues entre la colonne précédente 
et celle-ci; dans la lacune se trouvait précisément l'amorce de la citation. Elle figurait certainement dans un contexte où il était question de l'exil d'Euripide, mais il est impossible de savoir quel lien Satyros mettait entre l'acte malveillant qu'il rapporte et le voyage de 408 .

Ce qui est tout à fait caractéristique de la méthode de Satyros, c'est la citation qui se trouve à la fin de cette même colonne. "Voilà que des ailes d'or s'attachent à mon dos et, à mes pieds les plumes jointes des Sirènes; je marcherai vers le large éther pour m'unir à Zeus". C'est là, dit Satyros, une "récusation ", où Euripide abjure Athènes. Les vers étaient connus par une citation de Clément d'Alexandrie (fragm. 911) et Plutarque y fait allusion. Des déclarations de ce genre, on en trouverait des quantités dans Euripide, où, avec une fréquence raillée des comiques, un personnage souhaite avoir des ailes pour quitter au plus vite un monde où tout le dégoùte. Satyros, qui lisait tant d'œuvres d'Euripide que nous n'avons plus, était bien mieux placé que nous pour savoir que c'est là chez le poète le signe d'une tournure d'esprit et non l'annonce d'un authentique voyage. A la colonne suivante (XVIII), il interprète $Z_{\eta} \nu i \sigma v \mu \mu i \xi \omega \nu \delta \varrho \mu \tilde{a} v$ par « je vais accroître la puissance du roi ". On voit combien est dangereuse cette méthode qui essaye de découvrir dans les œuvres des renseignements biographiques et. dans ce que nous savons de la biographie, des moyens de trouver un second sens à des passages parfaitement clairs en soi.

La même colonne se termine par une erreur manifeste : qu'Euripide vieillit en Macédoine. Il y passa deux ans. La source de Satyros devait porter un terme général comme le $\delta \iota \varepsilon ́ \tau \varrho \iota \psi \varepsilon$ de Genos, 24, à quoi l'on pouvait se tromper. Encore faut-il ajouter que, pour s'y être trompé, Satyros devait n'avoir aucun intérêt pour la chronologie du poète auquel il consacrait son essai.Signalons en passant que c'est le seul indice de parenté entre Satyros et le début du Genos.

Les colonnes XIV à XVIII n'ont pas de correspondance dans le Genos, qui est plus court. On ne voit pas ce que l'auteur du Genos aurait trouvé à prendre dans ces citations de vers et ces exégèses à coups de citations. Ces passages intéressent plutôt la critique que l'histoire littéraire.

Vie, 39, XIX correspond à Genos 80-87. I ci et là, on veut montrer combien Euripide était admiré à l'étranger. Le Genos en donne 
comme preuve loffrande de Denys, rapportée par Hermippe. Satyros raconte l'histoire des prisonniers de Syracuse, qui sera reprise par Plutarque. On distingue dans ce fragment la composition soignée de Satyros : «Euripide, peu estimé des Athéniens de son temps, hommes et femmes, l'était des étrangers. Première preuve tirée des Siciliens. Seconde preuve, tirée des Macédoniens. Celle-ci, exposée dans la partie qui manque, servait de transition à l'histoire du $\sigma \tau o ́ \mu \alpha \delta v \sigma \tilde{\omega} \delta \varepsilon \varsigma$ qui se trouve dans les deux textes (Vie 39, XX et Genos 87-90).

L'anecdote est de celles qui sont faites pour amener la réponse. Ici, la réponse vient des vers d'Alexandre d'Étolie qu'Aulu-Gelle nous a conservés. Les voici : «Euripide, l'élève de l'antique Anaxagore, me paraît âpre à aborder; il hait le rire, même le vin ne lui enseigne point à plaisanter. Mais, ce qu'il écrit, cela est plus doux que le miel et les Sirènes ». Cela doit venir d'une comédie où Euripide est mis personnellement en scène et où le comique exagérait la rudesse d'abord du poète. Ces vers étaient célèbres, car la fin du premier se trouve dans notre Genos (67). On a dû être tenté assez tôt de les parodier en cherchant à doubler par les adjectifs l'antithèse

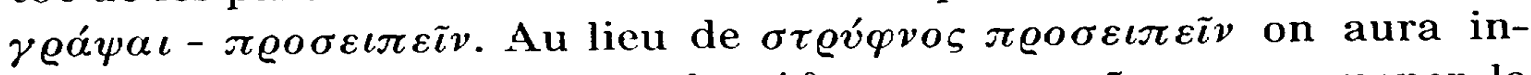
venté quelque chose comme $\delta v \sigma \omega ́ \delta \eta \varsigma \tau \varrho o \sigma \varepsilon \iota \pi \varepsilon i \bar{\nu}$ pour amener la

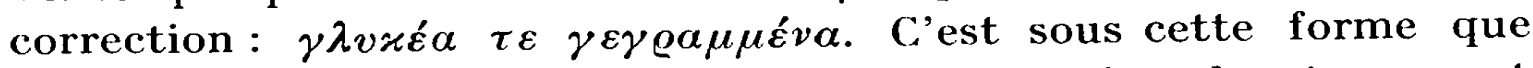
nous trouvons l'histoire dans Genos 88-90. Les interlocuteurs sont laissés dans le vague. Un jeune homme fait le reproche "par jalousie ". Il semble bien que ce soit Euripide qui réponde, par un texte un peu différent du vers d'Alexandre: l'altération prouve que l'anecdote avait déjà pas mal cheminé avant d'en arriver où nous la trouvons. Elle est restée purement littéraire : faite pour amener le trait final.

Ce qui est curieux, c'est qu'elle a perdu ce caractère sous la plume du très littéraire Satyros et qu'elle est devenue chez lui un potin de cour d'assez basse qualité. C'est un jeune amoureux d'Archélaos qui accuse Euripide d'avoir une mauvaise haleine et c'est Archélaos qui le rappelle au respect qu'il faut avoir pour une bouche d'où sont sortis tant de beaux vers et de beaux chants. Le vers d'Alexandre est complètement oublié et l'expression est maladroite et

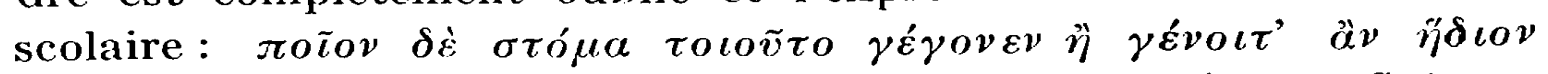

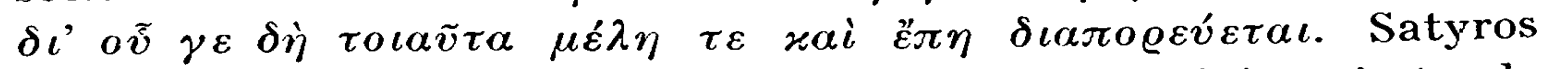
a dû trouver cette version dans la source qu'il a suivie; c'est cela 
qui lui a permis de la mettre dans le contexte macédonien. Dans le Genos, elle est détachée, puisque les interlocuteurs sont anonymes.

Vie, 39, XXI est à peu près mot pour mot l'équivalent de Genos, 49-61. C'est le récit de la mort d'Euripide lacéré par des chiens et l'histoire de la "vengeance de la chienne". Satyros prétend que cette absurdité était racontée par des "Macédoniens instruits et vénérables" (XX, 30-35). Ce n'est pas impossible. Il est probable qu'il y eut une atmosphère d'hostilité autour des poètes grecs qui entouraient le roi. Si vraiment Euripide est mort par accident - ce qui n'a rien d'invraisemblable - ses amis ont bien pu accuser des malveillants de l'avoir exposé ou de l'avoir laissé s'exposer au danger. Et l'on aura inventé en Macédoine des histoires destinées à innocenter en tous cas les gens du pays.

Arrivés à ce point, nous avons retrouvé dans la Vie toutes les anecdotes qui composent Genos II, ou des répliques de ces anecdotes, excepté les lignes 68-77 : mariage d'Euripide avec Choerilé, fille de Mnésiloque, qu'il répudie et qui se remarie. Il se remarie de son côté et n'est pas plus heureux. L'anecdote, avec l'allusion à Hippolyte voilé, était bien faite pour plaire à Satyros qui veut à tout prix trouver dans l'œuvre des allusions à la vie.Mais on ne voit pas bien dans quelle partie des fragments conservés elle pourrait se placer. Peut-être était-elle dans le début perdu. Peut-être aussi Satyros l'a-t-il à dessein laissée de côté dans une étude où Euripide a un beau rôle. Il est vrai qu'alors il eût dû ne pas reproduire l'histoire de Céphisophon.

Avec XXII commence un chapitre fort intéressant: rapports d'Euripide avec Timothée. Malheureusement, le papyrus s'arrête après 34 lignes de texte, qui nous donnent un renseignement probablement exact - Euripide s'intéressait aux innovations de Timothée - et un autre certainement inexact - il aurait collaboré au poème des Perses.

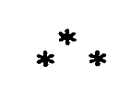

Après cette longue analyse, nous pouvons conclure.

La Vie de Satyros et le Genos II sont deux textes parallèles, contenant des anecdotes identiques ou équivalentes. Seulement, l'interprétation diffère: plus sarcastique dans le Genos, plus admirative dans Satyros. 
Il est possible que le rédacteur du Genos ait lu Satyros comme le veut $M$. Frey, mais je ne le pense pas. S'il avait eu sous les yeux le texte que le papyrus nous a rendu, il aurait donné une forme plus intelligible à l'anecdote de Céphisophon. La réponse sur la "bouche plus douce que le miel ", avec sa citation altérée d'un vieux poète, ne peut venir de Satyros. Si l'auteur de Genos II avait lu notre texte de la Vie, il aurait mis, comme elle, toute l'anecdote en rapport avec Archélaos.

Satyros a dû puiser dans un recueil d'anecdotes que tout le monde connaissait. Ces anecdotes, il les a commentées, étoffées de citations et de rapprochements littéraires. Il est ainsi amené à raconter, à propos d'elles, des faits sur l'authenticité desquels nous dirons un mot pour finir.

Le compilateur de Genos $I I$ doit avoir connu les mêmes anecdotes sous une forme plus brève, si brève qu'il ne les a plus toujours comprises. De plus, il leur a laissé tout leur décousu, tandis que Satyros les faisait entrer dans une composition très soignée. Le décousu du Genos a facilité toutes les interpolations ultérieures.Ainsi, l'hostilité des femmes est donnée deux fois (77-8 et 100-102); l'histoire de Céphisophon est donnée une fois comme une calomnie (78-80) et une fois comme un réalité (99 sqq.).

Il me paraît assez difficile d'admettre que si l'auteur de Genos II avait eu sous les yeux une étude aussi bien composée que celle de Satyros, il eût pu la reproduire dans le désordre où se trouve sa compilation.

Quel crédit mérite Satyros pour les faits nouveaux qu'il apporte? Leo estimait que les noms des poètes rivaux d'Euripide, Satyros pouvait les avoir trouvés dans un texte comique mais que, pour savoir qu'ils avaient concouru contre Euripide et qu'ils lui avaient été préférés, il avait dû lire des didascalies. Rien n'est moins sûr. De la plus vague allusion littéraire, Satyros tire des renseignements biographiques. Dans un passage lyrique où le chœur souhaite de s'envoler, il voit une annonce du départ macédonien; où Euripide dit Zeus, il entend Archélaos. S'il connaissait des textes comiques où l'on se moquait des succès immérités d'Acestor et des autres, il n'aura pas hésité à comparer ces couronnes aux échecs d'Euripi- 
de et cela, sans la moindre vérification. Nous ignorons du reste, quel passage comique est ici la source de Satyros. Acestor, "le Scythe ", le froid Morsimos, dont Suidas dit qu'il était médecin, sont souvent raillés par Aristophane, Cratinus et Eupolis. Morsinios et Mélanthios, "goinfres, plaie du marché au poisson, coureurs de vieilles", sont raillés ensemble dans la Paix (800 sqq) pour leurs échecs tragiques. I e passage de Satyros, sur leurs succès immérités, vient certainement d'ailleurs, d'un texte perdu où les quatre noms étaient rapprochés. Il semble bien que Satyros nous ait conservé la forme exacte du nom de Dorilaos (plutôt que Dorillos), sur lequel Aristophane jouait à cause de sa ressemblance avec $\delta$ o gía $\lambda \lambda \lambda_{5}$, pudendum muliebre.

En général, les rapprochements littéraires que donne Satyros sont intéressants, mais les faits qu'il est seul à mentionner sont fort sujets à caution. Qu'Euripide ait collaboré aux Perses de Timothée est tout à fait invraisemblable. Si Cléon avait intenté à Euripide un procès pour impiété, la chose aurait fait assez de bruit pour parvenir jusqu'à nous. De plus, un tel procès ne pourrait guère se placer qu'entre 429 et 422 . S'il avait eu lieu avant la mort de Périclès, il est probable que Plutarque le mentionnerait en même temps qu'il parle des procès de Phidias, d'Aspasie et d'Anaxagore. Entre la mort de Périclès et la mort de Cléon, Euripide prend à Athènes position de grand poète patriote. Il triomphe avec Hippolyte, écrit les Héraclides et Andromaque. Ce n'est pas le moment de s'attaquer à lui et Cléon, partisan de la guerre à outrance, y songera moins que personne. Il nous est impossible de savoir de quel passage littéraire Satyros aura déduit ce fait (1).

Ce qui nous rend prudents à l'endroit des faits nouveaux racontés par Satyros, c'est la qualité des anecdotes qu'il rapporte sans sourciller: Céphisophon, la conspiration des femmes, la vengeance des chiennes thraces, l'idée qu'Euripide aurait quitté Athènes par dépit. L'histoire des prisonniers de Syracuse ne se trouve pas avant Satyros: on ne voit pas comment il aurait pu l'inventer, sinon il faudrait également se méfier. Du reste, Plutarque qui raconte le fait dans la Vie de Nicias paraît avoir eu une source indépendante de Satyros, ce qui donne plus de poids à l'histoire.

(1) Au contraire, l'histoire racontée par Aristote (Rhét., III, 15, 8) d'un procès d'antidosis entre Euripide et Hygiénon parait bien authentique. 
Satyros a trouvé, pour écrire sa vie d'Euripide, un paquet d'anecdotes brèves et indigentes. Il avait une lecture énorme: presque tous les fragments qu'il apporte sont nouveaux pour nous, et nous avons essayé de montrer que ce n'est pas sa faute si ces fragments se présentent parfois dans le papyrus sous une forme incohérente. Dans toute son érudition, il se plâit visiblement à cueillir des citations peu connues. Le reste l'intéressait médiocrement.

Genos II vient du même paquet d'anecdotes. Le compilateur n'a pas même essayé de les mettre en ordre.

Genos $I$ et le chapitre des Nuits attiques viennent de collections analogues, la première presqu: exclusivement, la seconde, exclusivement biographique. Le texte d'Aulu-Gelle remonte à une tradition où l'enfance tenait une place prépondérante. Dans la notice de Suidas, c'est le roman macédonien qui est au premier plan.

La notice de Suidas, celle de Thomas Magister et la troisieme du Genos sont faites à l'usage des classes et composées avec soin, surtout la dernière. Toutes trois ont été écrites tard, à une époque où les discussions littéraires à coups de citations n'intéressaient plus guère. Toutes trois sont écrites sur un ton admiratif qui contraste avec le ton assez malveillant des notices biographiques et anecdotiques. Pour ce qui est du fond, elles doivent plus ou moins à Genos $I$ ou à sa source mais même Thomas Magister, qui est le plus proche de Genos $I$, contient des choses qui viennent d'ailleurs. Genos $I I I$ et Magister contiennent des appréciations littéraires; Suidas, seulement des renseignements littéraires.

Ainsi se groupent les biographies anciennes relatives à Euripide. Elles paraisscnt remonter toutes à des recueils d'anecdotes, plus ou moins intelligemment mises en ouvre. Parmi ces anecdotes subsistaient des traces de polémiques remontant à la critique aristotélicienne et alexandrine: Théopompe affirmant que la mère d'Euripide vendait des légumes, Philochore affirmant qu'elle était noble. Les notices composées de ces éléments disparates méritent une créance à peu près égale et très mesurée. Chaque renseignement, soigneusement pesé, se révèle bien léger. Déjà à l'époque de Satyros, on écrivait la vie d'Euripide d'après ses vers et d'après les moqueries des comiques. On partait d'un postulat: Euripide est un subjectif qui n'a pas pu s'exclure de son œuvre. Et le postulat est certes plus exact que les conséquences qu'on en a tirées. 